\title{
MULTIPLE-TRIAL CONFLICTS AND STOCHASTIC EVOLUTIONARY GAME DYNAMICS
}

\author{
LORENS A. IMHOF, ${ }^{*}$ Bonn University
}

\begin{abstract}
We consider stochastic replicator processes for games that are composed of finitely many trials. Several general results on the relation between Nash equilibria and the long-run behaviour of the stochastic processes are proved. In particular, a sufficient condition is given for almost sure convergence to a state where everyone plays in every trial a strict Nash equilibrium. The results are applied to multiple-trial conflicts based on wars of attrition and on sperm competition games with fair raffles, respectively.
\end{abstract}

Keywords: Nash equilibrium; random strategy; stochastic differential equation; stochastic replicator dynamics; war of attrition

2000 Mathematics Subject Classification: Primary 60J70

Secondary 91A22; 92D25

\section{Introduction}

This paper deals with a stochastic process that describes the evolution of a population under selection. The selection mechanism is based on a game which consists of a finite number of trials. A pure strategy in this game determines what to do in the individual trials. The members of the population are programmed to pure strategies and are paired at random to play the underlying multiple-trial game. The growth rate of a population share playing a particular strategy is given by the difference between the current payoff to that strategy and the average payoff to the population, where payoffs are subject to stochastic shocks. The aim of the present paper is to examine the long-run behaviour of the stochastic process.

We use a diffusion process, specifically, the stochastic replicator dynamics of Fudenberg and Harris (1992) to model the evolution of an infinite population. The precise formulation of the process is given in Section 2. Several general results for the stochastic replicator dynamics have been established recently. Cabrales (2000) studied extinction of iteratively strictly dominated strategies. Imhof (2005) gave a recurrence criterion and examined stationary distributions as well as local stability. Khasminskii and Potsepun (2006) analysed replicator dynamics under Stratonovich-type random perturbations. Hofbauer and Imhof (2007) considered time averages and provided some recurrence and transience criteria. The connection between recurrence and permanence of the deterministic analogue has been studied by Benaïm et al. (2008). Discrete stochastic replicator dynamics have been analysed by Benaïm et al. (2004) and Schreiber (2001).

The deterministic counterpart to the present stochastic process, the replicator dynamics of Taylor and Jonker (1978), has been extensively investigated and has found many diverse applications in biology and economics; see Cressman (1992), (2003), Hofbauer and Sigmund (1998), Weibull (1995), and the recent surveys by Hofbauer and Sigmund (2003) and Nowak and Sigmund (2004). The deterministic and the stochastic dynamics can behave quite differently,

Received 2 September 2005; revision received 27 July 2008.

* Postal address: Statistische Abteilung und Hausdorff-Zentrum für Mathematik, Universität Bonn, Adenauerallee 24-42, 53113 Bonn, Germany. Email address: limhof@uni-bonn.de 
though, even when the stochastic influences are small. For instance, only the states where the whole population uses the same strategy are stationary points of the stochastic dynamics, while every Nash equilibrium of the underlying game is a stationary point of the deterministic dynamics.

In Section 3 we deal with the game that drives the stochastic process and, in particular, with the corresponding Nash equilibria. Subsection 3.1 contains a brief review of the general model for multiple-trial conflicts developed by Whittaker and Cannings (1994). Particularly important to our analysis is their result which says that, under a certain definiteness condition, only random strategies need to be considered. We then focus on resource allocation games. Here every available strategy for the individual trials has a certain cost and the resources of the players are limited. In Subsection 3.2, the individual trials model wars of attrition, as already considered by Whittaker and Cannings (1994) and Whittaker (1996). The players distribute their resources to the trials and the winner of a trial is the player who has allocated the larger amount of resource to that trial. Whittaker (1996) gave an extensive analysis for the case where resources are relatively scarce. The main result of Subsection 3.2 substantiates a conjecture of Whittaker for the opposite case. In Subsection 3.3, the trials are sperm competition games with fair raffles, as in Parker (1990). Here two males mate with the same females. The males have to distribute a given amount of sperm to a fixed number of matings and in each mating the chance of fertilizing the female is proportional to the ratio of their own sperm to the total amount of sperm.

Section 4 builds on the setting and results of Section 3 and determines the long-run behaviour of the corresponding stochastic replicator dynamics. In Subsection 4.1, a general theorem is established which shows that the average distance between the stochastic process and a Nash equilibrium is small, provided that the payoff matrix satisfies a certain definiteness condition similar to that used by Whittaker and Cannings. This result is closely related to the folk theorem of deterministic evolutionary game theory. However, in the stochastic setting, the composition of the population need not converge towards a stable point even if there is a globally asymptotically stable state in the deterministic replicator dynamics. An application to a two-trial war of attrition shows that in the long run the population is composed mostly of players that use up all the resources available and distribute them unevenly over the two trials, provided the reward that can be obtained in the trials is large enough. The proportions of the population shares of the equilibrium are given explicitly in terms of Chebyshev polynomials. The frequency of players that allocate all resources to one trial is the largest and the frequency of those who distribute them nearly equally is the smallest positive one. As the value of the reward tends to $\infty$, the sizes of the shares become equal.

Subsection 4.2 is concerned with global asymptotic stability of strict Nash equilibria. First a general theorem, not restricted to the multiple-trial framework, is proved, which gives a sufficient condition for almost sure convergence to a strict Nash equilibrium for every initial state. An application of this result to multiple-trial conflicts shows that if the payoff matrix of the individual trials has a strict Nash equilibrium and satisfies a weak definiteness condition, then the stochastic replicator dynamics converge almost surely to a state where every member of the population uses the Nash equilibrium strategy in every trial. In the multiple-trial sperm competition game, it turns out that, generically, there is a certain optimal amount of sperm to be used in each round, and the population evolves to a state where all the males implement that optimal choice, provided that their resources are sufficient.

Subsection 4.3 is devoted to weakly dominated strategies. It is shown that, under the stochastic replicator dynamics, the frequency of a weakly dominated strategy is certain to 
fall below any positive threshold, which is not the case under the deterministic dynamics; see Weibull (1995, pp. 84-85). On the other hand, a weakly dominated strategy may persist in the stochastic model even if it becomes extinct in the deterministic model.

\section{The stochastic replicator dynamics}

Consider a symmetric two-player game with pure strategies $1, \ldots, n$ and payoff matrix $\boldsymbol{A}=\left(a_{j k}\right)$. Thus, $a_{j k}$ is the payoff to a player who uses strategy $j$ against an opponent who plays $k$. In the models to be discussed in the following sections, the game will be composed of a finite number of trials and the payoff $a_{j k}$ is the sum of the payoffs from the individual trials. In specifying the replicator dynamics, no assumptions on $\boldsymbol{A}$ are made. Consider a large population and suppose that every individual is programmed to play one fixed pure strategy. For every point of time $t \geq 0$, let $\zeta_{j}(t)$ be the size of the subpopulation of $j$-players. The population state is defined as $\xi(t)=\left(\xi_{1}(t), \ldots, \xi_{n}(t)\right)^{\top}$, where $\xi_{j}(t)=\zeta_{j}(t) /\left(\zeta_{1}(t)+\cdots+\zeta_{n}(t)\right)$ is the proportion of individuals in the population playing strategy $j$. The state space is given by

$$
\Delta=\left\{\boldsymbol{y} \in[0,1]^{n}: y_{1}+\cdots+y_{n}=1\right\} .
$$

For $j=1, \ldots, n$, let $\boldsymbol{e}_{j} \in \mathbb{R}^{n}$ be the $j$ th column of the unit matrix of order $n$. If the population state is $\boldsymbol{\xi}(t)$ and individuals are paired at random, then $\boldsymbol{e}_{j}^{\top} \boldsymbol{A} \boldsymbol{\xi}(t)$ is the average payoff to $j$-players. Suppose that the payoff represents the increase of fitness, measured as the number of offspring per unit of time, and suppose that strategies breed true. Then

$$
\frac{\mathrm{d} \zeta_{j}(t)}{\mathrm{d} t}=\zeta_{j}(t) \boldsymbol{e}_{j}^{\top} \boldsymbol{A} \boldsymbol{\xi}(t), \quad j=1, \ldots, n .
$$

Hence,

$$
\frac{\mathrm{d} \xi_{j}(t)}{\mathrm{d} t}=\xi_{j}(t)\left(\boldsymbol{e}_{j}^{\top} \boldsymbol{A} \boldsymbol{\xi}(t)-\boldsymbol{\xi}(t)^{\top} \boldsymbol{A} \boldsymbol{\xi}(t)\right), \quad j=1, \ldots, n .
$$

Equation (2.2) describes the deterministic replicator dynamics of Taylor and Jonker (1978); see Cressman (2003), Hofbauer and Sigmund (1998), and Weibull (1995) for more detailed explanations.

Following Fudenberg and Harris (1992), we now introduce stochastic shocks to the payoffs and consider the following variant of (2.1):

$$
\mathrm{d} Z_{j}(t)=Z_{j}(t)\left(\boldsymbol{e}_{j}^{\top} \boldsymbol{A} \boldsymbol{X}(t) \mathrm{d} t+\sigma_{j} \mathrm{~d} W_{j}(t)\right), \quad j=1, \ldots, n,
$$

where $\sigma_{1}, \ldots, \sigma_{n}$ are positive coefficients, $\left(W_{1}(t), \ldots, W_{n}(t)\right)^{\top}=\boldsymbol{W}(t)$ is an $n$-dimensional Brownian motion, and

$$
\boldsymbol{X}(t)=\left(X_{1}(t), \ldots, X_{n}(t)\right)^{\top}=\frac{1}{Z_{1}(t)+\cdots+Z_{n}(t)}\left(Z_{1}(t), \ldots, Z_{n}(t)\right)^{\top} .
$$

It follows that $\boldsymbol{X}(t)$ satisfies the stochastic differential equation

$$
\mathrm{d} \boldsymbol{X}(t)=\boldsymbol{a}(\boldsymbol{X}(t)) \mathrm{d} t+\boldsymbol{C}(\boldsymbol{X}(t)) \mathrm{d} \boldsymbol{W}(t)
$$

where

$$
\boldsymbol{a}(\boldsymbol{y})=\left[\operatorname{diag}\left(y_{1}, \ldots, y_{n}\right)-\boldsymbol{y} \boldsymbol{y}^{\top}\right]\left[\boldsymbol{A}-\operatorname{diag}\left(\sigma_{1}^{2}, \ldots, \sigma_{n}^{2}\right)\right] \boldsymbol{y}
$$

and

$$
\boldsymbol{C}(\boldsymbol{y})=\left[\operatorname{diag}\left(y_{1}, \ldots, y_{n}\right)-\boldsymbol{y} \boldsymbol{y}^{\top}\right] \operatorname{diag}\left(\sigma_{1}, \ldots, \sigma_{n}\right) .
$$


Equation (2.4) describes the stochastic replicator dynamics of Fudenberg and Harris (1992). For $\boldsymbol{x} \in \operatorname{int}(\Delta)=\left\{\boldsymbol{y} \in \Delta: y_{j}>0\right.$ for all $\left.j\right\}$, let $\mathrm{P}_{\boldsymbol{x}}$ denote the probability measure corresponding to the process $\boldsymbol{X}(t)$ with initial condition $\boldsymbol{X}(0)=\boldsymbol{x}$, and let $\mathrm{E}_{\boldsymbol{x}}$ denote expectation with respect to $\mathrm{P}_{\boldsymbol{x}}$. Note that

$$
\mathrm{P}_{\boldsymbol{x}}\{X(t) \in \operatorname{int}(\Delta) \text { for all } t \geq 0\}=1 \text { if } \boldsymbol{x} \in \operatorname{int}(\Delta) .
$$

Thus, the stochastic model (2.4) shares with the deterministic model (2.2) the property that none of the pure strategies that are initially present will become extinct in finite time. In the closely related stochastic model of Foster and Young (1990), however, the boundary may be crossed in finite time. They suggested introducing a reflecting boundary, which seems problematic in biological applications. Therefore, in the present paper, only model (2.4) will be considered.

\section{Multiple-trial conflicts}

To analyse, in the next section, the long-run behaviour of the stochastic evolutionary process, we first study stable strategies and equilibria of the game that drives the process. We focus on a class of games that model resource allocation problems in multiple-trial conflicts. A single game consists of a fixed number of trials; the players have a fixed amount of resources available and must decide how to allocate the resources to the individual trials. Multiple-trial conflicts are much more difficult to deal with than the corresponding single trials. This is because the number of pure strategies increases rapidly as the number of trials increases, and, more importantly, the structure of the payoff becomes more involved. Under certain conditions, a considerable simplification can be achieved by restricting attention to so-called random strategies. These are strategies which deliberately ignore the order of the trials. The present analysis relies on the framework developed by Whittaker and Cannings (1994). A brief description together with some extensions will be given in Subsection 3.1. The remainder of Section 3 is devoted to specific applications.

\subsection{A general model for multiple-trial conflicts}

Suppose that a single contest consists of $m$ trials, $m \geq 2$. Each of these trials is described by a symmetric two-player game with $v+1$ pure strategies, $0, \ldots, v$, and one-trial payoff matrix $\boldsymbol{A}^{(1)}=\left(a_{j k}^{(1)}\right)_{j, k=0}^{v}$. Playing strategy $j$ gives rise to a certain cost, say $c_{j} \geq 0$ units of resource. Each contestant has altogether $L$ units of resource available to allocate to the individual trials before the contest begins. Thus, the players are not allowed to base the choice of their strategies for the next trial on the outcome of previous trials. The set of pure strategies for the $m$-trial conflict is

$$
\mathbb{U}=\left\{\left(s_{1}, \ldots, s_{m}\right) \in\{0,1, \ldots, v\}^{m}: \sum_{\mu=1}^{m} c_{s_{\mu}} \leq L\right\},
$$

where $s_{\mu}$ is the strategy that is to be played in the $\mu$ th trial.

Let $\boldsymbol{r}, \boldsymbol{s} \in \mathbb{U}$. Then the $m$-trial payoff to an $\boldsymbol{r}$-player against an $\boldsymbol{s}$-player is $M(\boldsymbol{r}, \boldsymbol{s})=$ $\sum_{\mu=1}^{m} a_{r_{\mu}, s_{\mu}}^{(1)}$. Let $\mathcal{P}$ denote the set of all mixed strategies for the multiple-trial conflict, that is, $\mathcal{P}$ is the set of all probability measures on $\mathbb{U}$. For strategies $p, q \in \mathcal{P}$, let $M(p, q)$ be the payoff to a $p$-player against a $q$-player, that is,

$$
M(p, q)=\sum_{\boldsymbol{r} \in \mathbb{U}} \sum_{\boldsymbol{s} \in \mathbb{U}} p(\boldsymbol{r}) M(\boldsymbol{r}, \boldsymbol{s}) q(\boldsymbol{s}) .
$$


Strategies $p, q \in \mathcal{P}$ are said to form an evolutionarily neutral pair if

$$
M(p, p)=M(q, p) \quad \text { and } \quad M(p, q)=M(q, q) .
$$

Strategy $p \in \mathcal{P}$ is said to be evolutionarily stable against $q \in \mathcal{P}$ if either

$$
M(p, p)>M(q, p)
$$

or

$$
M(p, p)=M(q, p) \quad \text { and } \quad M(p, q)>M(q, q) .
$$

A nonempty set $\mathcal{Q} \subset \mathcal{P}$ is said to be an evolutionarily stable set (ES set) if every $p \in \mathcal{Q}$ is evolutionarily stable against every $q \in \mathcal{P} \backslash \mathcal{Q}$ and forms an evolutionarily neutral pair with every $q \in \mathbb{Q}$. Note that if an ES set contains exactly one strategy then this strategy is an evolutionarily stable strategy (ESS) in the sense of the definition of Maynard Smith and Price (1973).

For $p \in \mathcal{P}, k=0, \ldots, v$, and $\mu=1, \ldots, m$, let

$$
f_{k, \mu}(p)=\sum_{\substack{\boldsymbol{s} \in \mathbb{U} \\
s_{\mu}=k}} p(s), \quad \boldsymbol{f}_{\mu}(p)=\left(\begin{array}{c}
f_{0, \mu}(p) \\
\vdots \\
f_{v, \mu}(p)
\end{array}\right) .
$$

That is, if $p$ represents the frequencies with which the pure strategies in $\mathbb{U}$ occur in a population then $f_{k, \mu}(p)$ denotes the frequency with which the $k$ th strategy is used in the $\mu$ th trial. We have

$$
M(p, q)=\sum_{\mu=1}^{m} \boldsymbol{f}_{\mu}(p)^{\top} \boldsymbol{A}^{(1)} \boldsymbol{f}_{\mu}(q) .
$$

A strategy $p \in \mathcal{P}$ is said to be a random strategy if

$$
\boldsymbol{f}_{1}(p)=\boldsymbol{f}_{2}(p)=\cdots=\boldsymbol{f}_{m}(p) .
$$

Thus, for a random strategy, the probability of playing any fixed pure strategy $s \in\{0, \ldots, v\}$ is the same for all the $m$ trials. In this sense, a random strategy ignores the order of the trials. Note, however, that a random strategy does not necessarily assign the same probability to all rearrangements of a pure strategy $\left(s_{1}, \ldots, s_{m}\right) \in \mathbb{U}$. Consider, for example, a two-trial conflict with $v=2$, and suppose that

$$
p(0,1)=p(2,0)=p(1,2)=\frac{1}{3} .
$$

Then $f_{1}(p)=f_{2}(p)=\left(\frac{1}{3}, \frac{1}{3}, \frac{1}{3}\right)^{\top}$, so that $p$ is a random strategy, although $p(0,1)=\frac{1}{3} \neq$ $0=p(1,0)$.

A real $d \times d$ matrix $\boldsymbol{A}$ is said to be conditionally negative definite if

$$
\boldsymbol{y}^{\top} \boldsymbol{A} \boldsymbol{y}<0 \text { for all } \boldsymbol{y} \in \mathbb{R}^{d} \text { such that } y_{1}+\cdots+y_{d}=0 \text { and } \boldsymbol{y} \neq \mathbf{0} .
$$

If $\boldsymbol{y}^{\top} \boldsymbol{A} \boldsymbol{y} \leq 0$ for all $\boldsymbol{y}$ with $y_{1}+\cdots+y_{d}=0$ then $\boldsymbol{A}$ is said to be conditionally negative semidefinite.

Theorem 3.1. (Whittaker and Cannings (1994).) Suppose that the one-trial payoff matrix $\boldsymbol{A}^{(1)}$ is conditionally negative definite. Then the following holds. 
(a) The m-trial conflict has a unique ES set, say $\mathcal{Q}$. If $q$ is any member of $\mathcal{Q}$ then $\mathcal{Q}$ can be written as

$$
\mathcal{Q}=\left\{p \in \mathcal{P}: \boldsymbol{f}_{1}(p)=\cdots=\boldsymbol{f}_{m}(p)=\boldsymbol{f}_{1}(q)\right\} .
$$

In particular, the ES set contains only random strategies.

(b) If $p \in \mathcal{P}$ is a random strategy and

$$
M(q, p) \leq M(p, p) \text { for all random strategies } q \in \mathcal{P},
$$

then

$$
M(q, p) \leq M(p, p) \text { for all strategies } q \in \mathcal{P},
$$

that is, $p$ is a Nash equilibrium.

(c) A strategy belongs to the ES set if and only if it is a Nash equilibrium.

Theorem 3.1 will be applied to solve an allocation problem for the war of attrition game described in Subsection 3.2. In the sperm competition game of Subsection 3.3, the one-trial payoff matrix $\boldsymbol{A}^{(1)}$ is such that $\boldsymbol{z}^{\top} \boldsymbol{A}^{(1)} \boldsymbol{z}=0$ for all $\boldsymbol{z}$ with $z_{0}+\cdots+z_{v}=0$, so that Theorem 3.1 cannot be applied. The following extension covers this situation when the individual trials have a strict Nash equilibrium.

Theorem 3.2. Suppose that the one-trial payoff matrix $\boldsymbol{A}^{(1)}$ is conditionally negative semidefinite and that the pure strategy $k \in\{0, \ldots, \nu\}$ is a strict Nash equilibrium for $\boldsymbol{A}^{(1)}$. Suppose that $m c_{k} \leq L$. Let $p^{*} \in \mathcal{P}$ denote the $m$-trial strategy according to which $k$ is played in every trial. Then $p^{*}$ is the unique strict Nash equilibrium for the $m$-trial game. Moreover, there does not exist another strategy in $\mathcal{P}$ which forms an evolutionarily neutral pair with $p^{*}$ or is evolutionarily stable against $p^{*}$. In particular, $\left\{p^{*}\right\}$ is the unique ES set.

Proof. Since $k$ is a strict Nash equilibrium, $a_{j k}^{(1)}<a_{k k}^{(1)}$ for all $j \neq k$. Thus, for every $q \in \mathcal{P}$, by (3.1),

$$
M\left(q, p^{*}\right)=\sum_{\mu=1}^{m} \boldsymbol{f}_{\mu}(q)^{\top} \boldsymbol{A}^{(1)} \boldsymbol{f}_{\mu}\left(p^{*}\right)=\sum_{\mu=1}^{m} \boldsymbol{f}_{\mu}(q)^{\top}\left[a_{0 k}^{(1)}, \ldots, a_{\nu k}^{(1)}\right]^{\top} \leq m a_{k k}^{(1)}=M\left(p^{*}, p^{*}\right)
$$

and $M\left(q, p^{*}\right)=M\left(p^{*}, p^{*}\right)$ if and only if $q=p^{*}$. That is, $p^{*}$ is a strict Nash equilibrium. If $q \in \mathcal{P} \backslash\left\{p^{*}\right\}$ then

$$
\begin{aligned}
M(q, q)-M\left(p^{*}, q\right) & <M(q, q)-M\left(p^{*}, q\right)+M\left(p^{*}, p^{*}\right)-M\left(q, p^{*}\right) \\
& =\sum_{\mu=1}^{m}\left[\boldsymbol{f}_{\mu}(q)-\boldsymbol{f}_{\mu}\left(p^{*}\right)\right]^{\top} \boldsymbol{A}^{(1)}\left[\boldsymbol{f}_{\mu}(q)-\boldsymbol{f}_{\mu}\left(p^{*}\right)\right] \\
& \leq 0
\end{aligned}
$$

which completes the proof.

Example 3.1. The assertion of Theorem 3.1 need not hold when the one-trial payoff matrix is merely conditionally negative semidefinite. The matrix

$$
\boldsymbol{A}^{(1)}=\left(\begin{array}{ll}
a & b \\
a & b
\end{array}\right)
$$


is conditionally negative semidefinite and it is easily seen that in the corresponding two-trial conflict every pair of strategies is evolutionarily neutral. Thus, $\mathcal{P}$ is an ES set which contains nonrandom strategies.

\subsection{A two-trial war of attrition conflict}

Consider a two-trial conflict with one-trial payoff matrix $\boldsymbol{A}^{(1)}=\left(a_{j k}^{(1)}\right)$ given by

$$
a_{j k}^{(1)}=\left\{\begin{array}{ll}
-j, & j \leq k, \\
V-k, & j>k,
\end{array} \quad j, k=0, \ldots, L,\right.
$$

where $V>0$. This game is a discrete version of the war of attrition introduced by Maynard Smith and Price (1973). For a comprehensive discussion and extensions, see Bishop and Cannings (1978), and for the multiple-trial case, see Cannings and Whittaker (1995) and Whittaker (1996). Let $c_{j}=j$. Then the set of pure strategies for the two-trial conflict is

$$
\mathbb{U}=\left\{\left(s_{1}, s_{2}\right) \in\{0, \ldots, L\}^{2}: s_{1}+s_{2} \leq L\right\} .
$$

A typical interpretation is as follows. Each player chooses for each trial a strategy which determines the maximum amount of time for which he is prepared to fight or display. In each trial the fight ends when one of the players has reached his chosen limit, that player leaves, and the other one gains a reward of value $V$, which increases his fitness. The fitness of both players is reduced by an amount given by the length of the fight. Should both players leave simultaneously, neither obtains a reward. As pointed out by Whittaker and Cannings (1994), the multiple-trial war of attrition can also be regarded as a variant of the Colonel Blotto game (Karlin (1959, pp. 82-84)). Each player has $L$ regiments that he can assign to two posts. At each post, the player that has more regiments gains $V$ units and both players incur a loss given by the minimum number of regiments assigned to the post.

The matrix $\boldsymbol{A}^{(1)}$ is seen to be conditionally negative definite so that, in view of Theorem 3.1, only random strategies need to be considered as candidates for the unique ES set. The following theorem takes advantage of this fact and gives an explicit expression for the ES set under the condition that the reward $V$ is sufficiently large. It turns out that in this case, the ES set contains just a single strategy, an ESS. The result substantiates a conjecture of Whittaker (1996, Subsection 7.2) to the effect that the strategies in the ES set should use all of the resources available when the reward is large enough. It seems that, for smaller values of $V$, the structure of the ES set becomes rather more complicated.

Let $T_{j}(x)$ and $U_{j}(x)$ denote the Chebyshev polynomials of the first and second kind, respectively. Thus,

$$
T_{j}(x)=\cos (j \arccos x), \quad U_{j}(x)=\frac{1}{j+1} T_{j+1}^{\prime}(x), \quad j \in \mathbb{N}_{0} .
$$

Let $U_{-1}(x) \equiv 0$.

Theorem 3.3. Suppose that the one-trial payoff matrix is given by (3.2).

(a) Suppose that $L=2 K+1, K \in \mathbb{N}_{0}$. If

$$
V>U_{K}\left(1+\frac{1}{V}\right)
$$


then the two-trial conflict has a unique ESS, namely the random strategy $p$ with

$$
\begin{aligned}
p(k, L-k) & =p(L-k, k) \\
& =\frac{U_{K-k}\left(1+V^{-1}\right)-U_{K-k-1}\left(1+V^{-1}\right)}{2 U_{K}\left(1+V^{-1}\right)}, \quad k=0,1 \ldots, K, \\
p(s) & =0 \text { for all } s \in \mathbb{U} \backslash\{(0, L),(1, L-1), \ldots,(L, 0)\} .
\end{aligned}
$$

(b) Suppose that $L=2 K, K \in \mathbb{N}$. If

$$
V>\frac{U_{K-1}\left(1+V^{-1}\right)+U_{K}\left(1+V^{-1}\right)-1}{2}
$$

then the two-trial conflict has a unique ESS, namely the random strategy $p$ with

$$
\begin{gathered}
p(k, L-k)=p(L-k, k)=\frac{T_{K-k}\left(1+V^{-1}\right)}{U_{K-1}\left(1+V^{-1}\right)+U_{K}\left(1+V^{-1}\right)}, \quad k=0,1 \ldots, K, \\
p(s)=0 \quad \text { for all } s \in \mathbb{U} \backslash\{(0, L),(1, L-1), \ldots,(L, 0)\} .
\end{gathered}
$$

The proof of Theorem 3.3 is given in Appendix A. Some interesting properties of the ESS $p$ can be derived from the explicit expressions; see Theorem 4.3, below.

\subsection{A multiple-trial sperm competition conflict}

Sperm competition games model competition between ejaculation strategies of males over fertilization when several males may mate with the same females. For each male, the probability of successful fertilization increases with the proportion of his sperm in the female tract. Here the case where two males mate with a fixed number of females will be analysed in the above general framework for multiple-trial conflicts. The individual trials are modelled by a discrete version of the sperm competition games with fair raffles described by Parker (1990).

Suppose that the $v+1$ pure strategies, $0,1, \ldots, v$, correspond to the possible ejaculate volumes $(0 \leq) \eta_{0}<\cdots<\eta_{v}$ with associated costs $(0 \leq) c_{0}<c_{1}<\cdots<c_{\nu}$. Suppose further that

$$
\frac{c_{j}-c_{j-1}}{\eta_{j}-\eta_{j-1}} \leq \frac{c_{j+1}-c_{j}}{\eta_{j+1}-\eta_{j}}, \quad j=1, \ldots, v-1 .
$$

Condition (3.6) means that the cost per additional unit of sperm does not decrease when the total amount used at a single trial increases. Suppose that, over the range of possible ejaculate volumes $\eta$ in the female tract, that is, for $\eta \in\left[2 \eta_{0}, 2 \eta_{\nu}\right]$, the probability $\phi(\eta)$ of fertilization after mating is either constant, $\phi(\eta)=\alpha>0$, or is given by an increasing affine function, $\phi(\eta)=\alpha+\beta \eta$, where $\alpha \geq 0$ and $\beta>0$. If $\eta_{0}=0$, let $\alpha=0$ and $\beta>0$. Sperm competition is assumed to obey the fair raffle principle. Thus, in the case of fertilization, the success probability of a $j$-player in competition with a $k$-player is equal to $\eta_{j} /\left(\eta_{j}+\eta_{k}\right)$. The expected gain of the $j$-player is therefore $\left(\eta_{j} /\left(\eta_{j}+\eta_{k}\right)\right) \phi\left(\eta_{j}+\eta_{k}\right) V$, where $V>0$ denotes the value of fertilization to the successful male. This gives rise the following one-trial payoffs:

$$
a_{j k}^{(1)}=\frac{\eta_{j}}{\eta_{j}+\eta_{k}} \phi\left(\eta_{j}+\eta_{k}\right) V-c_{j}, \quad j, k=0, \ldots, \nu .
$$

If $\eta_{0}=0$, set $a_{00}^{(1)}=-c_{0}$.

Write

$$
\rho_{k}=2 \frac{c_{k+1}-c_{k}}{\eta_{k+1}-\eta_{k}}\left(\frac{\phi\left(\eta_{k}+\eta_{k+1}\right)}{\eta_{k}+\eta_{k+1}}+\beta\right)^{-1}, \quad k=0, \ldots, v-1
$$


and $\rho_{-1}=0, \rho_{v}=\infty$. If $\alpha>0$, it follows from (3.6) that

$$
\rho_{-1}<\rho_{0}<\rho_{1}<\cdots<\rho_{\nu} .
$$

If $\alpha=0$, assume for simplicity that the inequalities in (3.6) are strict, so that (3.8) continues to hold. The next theorem gives the ES sets for the $m$-trial conflict under this assumption. It would not be very difficult, but technically somewhat cumbersome, to extend the theorem to the case where some of the inequalities in (3.8) degenerate to equalities. The proof of Theorem 3.4 is given in Appendix B.

Theorem 3.4. The matrix $\boldsymbol{A}^{(1)}$ given by (3.7) is conditionally negative semidefinite. If $V \in$ $(0, \infty) \backslash\left\{\rho_{0}, \ldots, \rho_{\nu-1}\right\}$ then there is a unique $k \in\{0, \ldots, v\}$ such that

$$
\rho_{k-1}<V<\rho_{k},
$$

strategy $k$ is a strict Nash equilibrium in each trial, and the unique ES set for the $m$-trial conflict consists of the probability measure that assigns mass 1 to $(k, k, \ldots, k)$, provided that $m c_{k} \leq L$.

If $V=\rho_{k}$ for some $k \in\{0, \ldots, v-1\}$ then the unique ES set consists of all the probability measures in $\mathcal{P}$ that assign mass 1 to $\{k, k+1\}^{m}$, provided that $m c_{k} \leq L$.

\section{Long-run behaviour of the stochastic dynamics}

\subsection{Nash equilibria}

According to the folk theorem of evolutionary game theory, an asymptotically stable point of the deterministic replicator dynamics, (2.2), must be a Nash equilibrium of $\boldsymbol{A}$. However, a Nash equilibrium is not necessarily stable. The next theorem gives a sufficient condition on the payoff matrix which ensures that the expected distance between the stochastic process $X(t)$ and a Nash equilibrium $\boldsymbol{p}$ is small. An upper bound is established for the expected time average

$$
\mathrm{E}_{\boldsymbol{x}} \frac{1}{t} \int_{0}^{t}\|\boldsymbol{X}(s)-\boldsymbol{p}\|^{2} \mathrm{~d} s
$$

where $\|\cdot\|$ denotes the Euclidean norm. When $t$ is large, the bound is of the order of magnitude of $\sigma_{1}^{2}, \ldots, \sigma_{n}^{2}$. It will be shown in Theorem 4.4, below, that (4.1) is in fact bounded away from 0 unless $\boldsymbol{p}$ is a pure strategy.

Let $\operatorname{supp}(\boldsymbol{p})=\left\{j: p_{j}>0\right\}$.

Theorem 4.1. Let $X(t)$ be given by the stochastic replicator dynamics (2.4) with initial condition $\boldsymbol{X}(0)=\boldsymbol{x} \in \operatorname{int}(\Delta)$. Let $\boldsymbol{p} \in \Delta$ be a Nash equilibrium for the underlying payoff matrix $\boldsymbol{A}$. Suppose that $\boldsymbol{A}$ satisfies the strict inequality

$$
\boldsymbol{z}^{\top} \boldsymbol{A} \boldsymbol{z}<0 \text { for all } \boldsymbol{z} \in \mathcal{Z}
$$

where

$$
\mathcal{Z}=\left\{z \in \mathbb{R}^{n}: z \neq \mathbf{0}, z_{1}+\cdots+z_{n}=0, z_{j} \geq 0 \text { for all } j \in\{1, \ldots, n\} \backslash \operatorname{supp}(\boldsymbol{p})\right\} .
$$

Then, for every $t>0$,

$$
\mathrm{E}_{\boldsymbol{x}} \frac{1}{t} \int_{0}^{t}\|\boldsymbol{X}(s)-\boldsymbol{p}\|^{2} \mathrm{~d} s \leq \frac{1}{|\lambda|}\left(\frac{d(\boldsymbol{x}, \boldsymbol{p})}{t}-\frac{1}{2 \sum_{j=1}^{n} \sigma_{j}^{-2}}+\frac{1}{2} \sum_{j=1}^{n} p_{j} \sigma_{j}^{2}\right),
$$


where $d(\boldsymbol{x}, \boldsymbol{p})=\sum_{j: p_{j}>0} p_{j} \log \left(p_{j} / x_{j}\right)$ is the Kullback-Leibler distance and

$$
\lambda=\max \left\{\boldsymbol{z}^{\top} \boldsymbol{A} z: z \in \mathcal{Z}, \boldsymbol{z}^{\top} \boldsymbol{z}=1\right\} .
$$

Proof. The argument is a suitable modification of the method of Lyapunov functions as described in Skorokhod (1989, Chapter III, Section 1). Note that, under the present conditions, $\boldsymbol{p}$ will in general not be a stationary point.

Fix any initial value $\boldsymbol{x} \in \operatorname{int}(\Delta)$. Set $g(\boldsymbol{y})=\sum_{j: p_{j}>0} p_{j} \log \left(p_{j} / y_{j}\right)$ for all $\boldsymbol{y} \in \operatorname{int}(\Delta)$. For $K>g(\boldsymbol{x})$, let $\tau_{K}=\inf \{t>0: g(\boldsymbol{X}(t))=K\}$. Then, by Dynkin's formula,

$$
0 \leq \mathrm{E}_{\boldsymbol{x}} g\left(\boldsymbol{X}\left(t \wedge \tau_{K}\right)\right)=g(\boldsymbol{x})+\mathrm{E}_{\boldsymbol{x}} \int_{0}^{t \wedge \tau_{K}} \mathcal{A} g(\boldsymbol{X}(s)) \mathrm{d} s,
$$

where the differential operator $\mathcal{A}$ is given by

$$
\mathcal{A} h=(\nabla h)^{\top} \boldsymbol{a}+\frac{1}{2} \operatorname{tr}\left(\nabla^{2} h \boldsymbol{C} \boldsymbol{C}^{\top}\right)
$$

with

$$
\boldsymbol{a}(\boldsymbol{y})=\left[\operatorname{diag}\left(y_{1}, \ldots, y_{n}\right)-\boldsymbol{y} \boldsymbol{y}^{\top}\right]\left[\boldsymbol{A}-\operatorname{diag}\left(\sigma_{1}^{2}, \ldots, \sigma_{n}^{2}\right)\right] \boldsymbol{y}
$$

and

$$
\boldsymbol{C}(\boldsymbol{y})=\left[\operatorname{diag}\left(y_{1}, \ldots, y_{n}\right)-\boldsymbol{y} \boldsymbol{y}^{\top}\right] \operatorname{diag}\left(\sigma_{1}, \ldots, \sigma_{n}\right) .
$$

Now

$$
\nabla g(\boldsymbol{y})=\left(-\frac{p_{1}}{y_{1}}, \ldots,-\frac{p_{n}}{y_{n}}\right)^{\top}, \quad \nabla^{2} g(\boldsymbol{y})=\operatorname{diag}\left(\frac{p_{1}}{y_{1}^{2}}, \ldots, \frac{p_{n}}{y_{n}^{2}}\right)
$$

and so

$$
\begin{aligned}
\mathscr{A} g(\boldsymbol{y}) & =(\boldsymbol{y}-\boldsymbol{p})^{\top}\left[\boldsymbol{A}-\operatorname{diag}\left(\sigma_{1}^{2}, \ldots, \sigma_{n}^{2}\right)\right] \boldsymbol{y}+\frac{1}{2} \sum_{j=1}^{n}\left(p_{j}+y_{j}^{2}-2 y_{j} p_{j}\right) \sigma_{j}^{2} \\
& =(\boldsymbol{y}-\boldsymbol{p})^{\top} \boldsymbol{A} \boldsymbol{y}-\frac{1}{2} \sum_{j=1}^{n} y_{j}^{2} \sigma_{j}^{2}+\frac{1}{2} \sum_{j=1}^{n} p_{j} \sigma_{j}^{2} .
\end{aligned}
$$

By the Cauchy-Schwarz inequality,

$$
1=\left(\sum_{j=1}^{n} y_{j} \sigma_{j} \sigma_{j}^{-1}\right)^{2} \leq \sum_{j=1}^{n} y_{j}^{2} \sigma_{j}^{2} \sum_{j=1}^{n} \sigma_{j}^{-2},
$$

so that $-\sum_{j=1}^{n} y_{j}^{2} \sigma_{j}^{2} \leq-\left(\sum_{j=1}^{n} \sigma_{j}^{-2}\right)^{-1}$. As $\boldsymbol{p}$ is a Nash equilibrium, $(\boldsymbol{y}-\boldsymbol{p})^{\top} \boldsymbol{A} \boldsymbol{p} \leq 0$. Moreover, $\boldsymbol{y}-\boldsymbol{p} \in \mathcal{Z} \cup\{\boldsymbol{0}\}$. Therefore,

$$
(\boldsymbol{y}-\boldsymbol{p})^{\top} \boldsymbol{A} \boldsymbol{y} \leq(\boldsymbol{y}-\boldsymbol{p})^{\top} \boldsymbol{A}(\boldsymbol{y}-\boldsymbol{p}) \leq \lambda\|\boldsymbol{y}-\boldsymbol{p}\|^{2} .
$$

It now follows that

$$
\begin{aligned}
0 \leq & g(\boldsymbol{x})-|\lambda| \mathrm{E}_{\boldsymbol{x}} \int_{0}^{t \wedge \tau_{K}}\|\boldsymbol{X}(s)-\boldsymbol{p}\|^{2} \mathrm{~d} s \\
& +\left(-\frac{1}{2 \sum_{j=1}^{n} \sigma_{j}^{-2}}+\frac{1}{2} \sum_{j=1}^{n} p_{j} \sigma_{j}^{2}\right) \mathrm{E}_{\boldsymbol{x}}\left(t \wedge \tau_{K}\right) .
\end{aligned}
$$

If $K \rightarrow \infty$ then $t \wedge \tau_{K} \rightarrow t$, and the assertion follows by the dominated convergence theorem. 
Remark 4.1. Under the assumptions of Theorem 4.1, $\boldsymbol{p}$ is the unique Nash equilibrium. To see this, suppose that $\boldsymbol{q}$ is another Nash equilibrium and set $\boldsymbol{z}=\boldsymbol{q}-\boldsymbol{p}$. Then $\boldsymbol{p}^{\top} \boldsymbol{A} \boldsymbol{p} \geq \boldsymbol{q}^{\top} \boldsymbol{A} \boldsymbol{p}$ and $\boldsymbol{q}^{\top} \boldsymbol{A} \boldsymbol{q} \geq \boldsymbol{p}^{\top} \boldsymbol{A} \boldsymbol{q}$, so that $\boldsymbol{z}^{\top} \boldsymbol{A} \boldsymbol{z} \geq 0$. If $\boldsymbol{q} \neq \boldsymbol{p}$ then $\boldsymbol{z} \in \mathcal{Z}$, and so, by (4.2), $\boldsymbol{z}^{\top} \boldsymbol{A} \boldsymbol{z}<0$. It follows that $\boldsymbol{q}=\boldsymbol{p}$.

We now return to the two-trial conflict of Subsection 3.2 based on the war of attrition given by (3.2) with reward $V$. Fix $L$, the number of units of resource available to each player. Denote the pure strategies in $\mathbb{U}=\left\{\left(s_{1}, s_{2}\right) \in\{0, \ldots, L\}: s_{1}+s_{2} \leq L\right\}$ by $1, \ldots, n$. Thus, the indices $1, \ldots, n$ of the components of vectors in $\Delta \subset \mathbb{R}^{n}$ now correspond to the elements of $\mathbb{U}$ in a fixed order. This yields an obvious one-to-one relation between the points in $\Delta$ and the mixed strategies in $\mathcal{P}$.

Theorem 4.2. Let $\boldsymbol{X}(t)$ be given by the stochastic replicator dynamics (2.4) for the two-trial war of attrition game described in Subsection 3.2. Suppose that the reward $V$ satisfies the condition of Theorem 3.3. Let $p \in \mathcal{P}$ be the random strategy defined in Theorem 3.3, and let $\boldsymbol{p}$ denote the corresponding point in $\Delta$. Then $\boldsymbol{X}(t)$ satisfies (4.3). In particular, for every $\boldsymbol{x} \in \operatorname{int}(\Delta)$,

$$
\limsup _{t \rightarrow \infty} \frac{1}{t} \mathrm{E}_{\boldsymbol{x}} \int_{0}^{t}\|\boldsymbol{X}(s)-\boldsymbol{p}\|^{2} \mathrm{~d} s \leq \frac{1}{2|\lambda|} \sum_{j=1}^{n} p_{j} \sigma_{j}^{2},
$$

where $\lambda$ is given by (4.4).

Proof. By Theorem 3.3, $\boldsymbol{p}$ is an ESS and, in particular, a Nash equilibrium. Thus, the assertion follows from Theorem 4.1, provided that condition (4.2) is met. That is, using the notation from Subsection 3.1, we have to prove that

$$
G(z)<0 \text { for all } z \in \mathbb{Z}
$$

where, for $z: \mathbb{U} \rightarrow \mathbb{R}$,

$$
G(z)=\sum_{\boldsymbol{r} \in \mathbb{U}} \sum_{\boldsymbol{s} \in \mathbb{U}} z(\boldsymbol{r}) M(\boldsymbol{r}, \boldsymbol{s}) z(\boldsymbol{s})
$$

and

$$
\mathcal{Z}=\left\{z: \sum_{\boldsymbol{s} \in \mathbb{U}} z(s)=0, z(s) \neq 0 \text { for some } s \in \mathbb{U}, z\left(s_{1}, s_{2}\right) \geq 0 \text { whenever } s_{1}+s_{2}<L\right\} .
$$

Let $z: \mathbb{U} \rightarrow \mathbb{R}$ be such that $\sum_{s \in \mathbb{U}} z(s)=0$. Then, since $A^{(1)}$ is conditionally strictly negative definite (Whittaker and Cannings (1994, proof of Theorem 5.1.1)),

$$
\begin{aligned}
G(z)= & \sum_{\left(r_{1}, r_{2}\right) \in \mathbb{U}} \sum_{\left(s_{1}, s_{2}\right) \in \mathbb{U}} z\left(r_{1}, r_{2}\right)\left(a_{r_{1}, s_{1}}^{(1)}+a_{r_{2}, s_{2}}^{(1)}\right) z\left(s_{1}, s_{2}\right) \\
= & \sum_{r_{1}=0}^{L} \sum_{s_{1}=0}^{L}\left(\sum_{r_{2}=0}^{L-r_{1}} z\left(r_{1}, r_{2}\right)\right) a_{r_{1}, s_{1}}^{(1)}\left(\sum_{s_{2}=0}^{L-s_{1}} z\left(s_{1}, s_{2}\right)\right) \\
& +\sum_{r_{2}=0}^{L} \sum_{s_{2}=0}^{L}\left(\sum_{r_{1}=0}^{L-r_{2}} z\left(r_{1}, r_{2}\right)\right) a_{r_{2}, s_{2}}^{(1)}\left(\sum_{s_{1}=0}^{L-s_{2}} z\left(s_{1}, s_{2}\right)\right) \\
\leq & 0 .
\end{aligned}
$$


Moreover, $G(z)=0$ if and only if

$$
\sum_{s_{2}=0}^{L-s_{1}} z\left(s_{1}, s_{2}\right)=0 \quad \text { for all } s_{1}=0, \ldots, L
$$

and

$$
\sum_{s_{1}=0}^{L-s_{2}} z\left(s_{1}, s_{2}\right)=0 \quad \text { for all } s_{2}=0, \ldots, L .
$$

Now assume that $z \in \mathcal{Z}$ and $G(z)=0$. It will be shown by induction that, for $s_{1}=0, \ldots, L$,

$$
z\left(s_{1}, s_{2}\right)=0 \quad \text { for } 0 \leq s_{2} \leq L-s_{1} .
$$

Clearly, this would mean that $z \equiv 0$, contradicting the assumption that $z \in \mathcal{Z}$, so that (4.6) must hold. By $(4.8), z(0, L)=0$. Since $z\left(0, s_{2}\right) \geq 0$ for $0 \leq s_{2}<L$, and since, by (4.7), $\sum_{s_{2}=0}^{L} z\left(0, s_{2}\right)=0$, it follows that (4.9) holds for $s_{1}=0$. Assume that (4.9) holds for $s_{1}=0, \ldots, S-1$, where $1 \leq S \leq L$. Then, by (4.8), $z(S, L-S)=\sum_{S_{1}=0}^{S} z\left(s_{1}, L-S\right)=0$. Again, since $z\left(S, s_{2}\right) \geq 0$ for $0 \leq s_{2}<L-S$, and since, by (4.7), $\sum_{s_{2}=0}^{L-S} z\left(S, s_{2}\right)=0$, it follows that (4.9) holds for $s_{1}=S$.

Remark 4.2. Condition (4.2) is weaker than the condition that the payoff matrix be conditionally negative definite. Indeed, the weaker condition is satisfied in the two-trial game just considered, but the stronger one is not. In particular, the results of Imhof (2005) are not applicable here.

Since, in view of Theorem 4.2, the random strategy $p$ gives an accurate description of the long-run behaviour of the process $\boldsymbol{X}(t)$ when $\sigma_{1}, \ldots, \sigma_{n}$ are small, it seems worthwhile to record some properties of $p$. Obviously, $p$ concentrates on strategies that make use of all available resources and it is indeed plausible that only individuals that are willing to invest all resources play a significant role in the population, provided that the reward of winning a trial is large enough. The next theorem shows in particular that $p$ assigns the largest probability to the strategies that allocate all resources to one of the two trials and the least positive probability to the strategies that distribute the resources (nearly) evenly. Moreover, as $V \rightarrow \infty, p$ converges weakly to the uniform distribution on the strategies that use all resources.

Theorem 4.3. If $p$ is the random strategy from Theorem 4.2 then

$$
p(0, L)>p(1, L-1)>\cdots>p\left(\left\lfloor\frac{L}{2}\right\rfloor,\left\lfloor\frac{L+1}{2}\right\rfloor\right),
$$

where $\lfloor x\rfloor$ denotes the greatest integer less than or equal to $x$, and

$$
\lim _{V \rightarrow \infty} p(k, L-k)=\frac{1}{L+1}, \quad k=0, \ldots, L .
$$

Proof. Suppose that $L=2 K+1$. Inequalities (4.10) follow from the strict total positivity of ultraspherical polynomials; see Karlin (1968, p. 444). Set $\bar{u}_{k}=U_{k}\left(1+V^{-1}\right) /(k+1)$. Then the kernel $\left(\bar{u}_{k+j}\right)$ is totally positive, which means in particular that

$$
\bar{u}_{k}<\sqrt{\bar{u}_{k-1} \bar{u}_{k+1}} \leq \frac{\bar{u}_{k-1}+\bar{u}_{k+1}}{2}, \quad \bar{u}_{k-1}<\bar{u}_{k}<\bar{u}_{k+1},
$$


so that

$$
\begin{aligned}
U_{k+1} & \left(1+V^{-1}\right)-2 U_{k}\left(1+V^{-1}\right)+U_{k-1}\left(1+V^{-1}\right) \\
& =(k+1)\left(\bar{u}_{k+1}-2 \bar{u}_{k}+u_{k-1}\right)+\bar{u}_{k+1}-\bar{u}_{k-1} \\
& >0
\end{aligned}
$$

The claimed inequalities (4.10) are now obvious from (3.4). Moreover, for $k=0, \ldots, K$,

$$
\lim _{V \rightarrow \infty} p(k, L-k)=\lim _{V \rightarrow \infty} p(L-k, k)=\frac{U_{K-k}(1)-U_{K-k-1}(1)}{2 U_{K}(1)}=\frac{1}{2(K+1)} .
$$

The arguments are similar for $L=2 K$.

Theorem 4.1 raises the question of whether it is possible to show that

$$
\mathrm{E}_{\boldsymbol{x}} \frac{1}{t} \int_{0}^{t}\|\boldsymbol{X}(s)-\boldsymbol{p}\|^{2} \mathrm{~d} s \rightarrow 0 \quad \text { as } t \rightarrow \infty,
$$

perhaps by choosing a better point $\boldsymbol{p}$ or by imposing further conditions on $\boldsymbol{p}$ and $\boldsymbol{A}$. The next theorem states that this is not possible, unless $\boldsymbol{p}$ is a pure strategy.

Theorem 4.4. Let $\boldsymbol{A}$ be an arbitrary payoff matrix, and let $\boldsymbol{X}(t)$ be given by the stochastic replicator dynamics (2.4) with initial condition $\boldsymbol{X}(0)=\boldsymbol{x} \in \operatorname{int}(\Delta)$. Let $\boldsymbol{p} \in \Delta$ be such that $|\operatorname{supp}(\boldsymbol{p})| \geq 2$. Then

$$
\liminf _{t \rightarrow \infty} \mathrm{E}_{\boldsymbol{x}} \frac{1}{t} \int_{0}^{t}\|\boldsymbol{X}(s)-\boldsymbol{p}\|^{2} \mathrm{~d} s>0 .
$$

Proof. Let $g \in C^{2}(\Delta)$ be such that $g(\boldsymbol{y})=\sum_{j: p_{j}>0} p_{j} \log \left(p_{j} / y_{j}\right)$ for all $\boldsymbol{y}$ in an open neighbourhood $U$ of $\boldsymbol{p}$. Let $\mathcal{A}$ be defined by (4.5). For $\boldsymbol{y} \in U$, we have, as in the proof of Theorem 4.1,

$$
\mathcal{A} g(\boldsymbol{y})=(\boldsymbol{y}-\boldsymbol{p})^{\top} \boldsymbol{A} \boldsymbol{y}-\frac{1}{2} \sum_{j=1}^{n} y_{j}^{2} \sigma_{j}^{2}+\frac{1}{2} \sum_{j=1}^{n} p_{j} \sigma_{j}^{2},
$$

so that

$$
\mathcal{A} g(\boldsymbol{p})=\frac{1}{2} \sum_{j=1}^{n} p_{j}\left(1-p_{j}\right) \sigma_{j}^{2}=: \gamma_{1}
$$

say. Note that $\gamma_{1}>0$, since $|\operatorname{supp}(\boldsymbol{p})| \geq 2$. Thus, there exists some $\gamma_{2}>0$ such that

$$
\mathcal{A} g(\boldsymbol{y})>\frac{\gamma_{1}}{2} \quad \text { if }\|\boldsymbol{y}-\boldsymbol{p}\|^{2}<\gamma_{2}
$$

Set

$$
\gamma_{3}=\max \{0, \max \{-\mathcal{A} g(\boldsymbol{y}): \boldsymbol{y} \in \Delta\}\}, \quad \gamma_{4}=\max \{g(\boldsymbol{y}): \boldsymbol{y} \in \Delta\} .
$$

Then

$$
\mathcal{A} g(\boldsymbol{y}) \geq \frac{\gamma_{1}}{2}-\frac{\gamma_{1}+2 \gamma_{3}}{2 \gamma_{2}}\|\boldsymbol{y}-\boldsymbol{p}\|^{2} \quad \text { for all } \boldsymbol{y} \in \Delta,
$$

and so, by Dynkin's formula,

$$
\begin{aligned}
\gamma_{4} & \geq \mathrm{E}_{\boldsymbol{x}} g(\boldsymbol{X}(t)) \\
& =g(\boldsymbol{x})+\mathrm{E}_{\boldsymbol{x}} \int_{0}^{t} \mathcal{A} g(\boldsymbol{X}(s)) \mathrm{d} s \\
& \geq g(\boldsymbol{x})+\frac{\gamma_{1} t}{2}-\frac{\gamma_{1}+2 \gamma_{3}}{2 \gamma_{2}} \mathrm{E}_{\boldsymbol{x}} \int_{0}^{t}\|\boldsymbol{X}(s)-\boldsymbol{p}\|^{2} \mathrm{~d} s \quad \text { for all } t>0 .
\end{aligned}
$$


Hence,

$$
\begin{aligned}
\liminf _{t \rightarrow \infty} \frac{1}{t} \mathrm{E}_{\boldsymbol{x}} \int_{0}^{t}\|\boldsymbol{X}(s)-\boldsymbol{p}\|^{2} \mathrm{~d} s & \geq \frac{2 \gamma_{2}}{\gamma_{1}+2 \gamma_{3}}\left(\lim _{t \rightarrow \infty} \frac{g(\boldsymbol{x})-\gamma_{4}}{t}+\frac{\gamma_{1}}{2}\right) \\
& =\frac{\gamma_{1} \gamma_{2}}{\gamma_{2}+2 \gamma_{3}} \\
& >0 .
\end{aligned}
$$

\subsection{Strict Nash equilibria}

Consider a multiple-trial game where the one-trial payoff matrix has a unique strict Nash equilibrium, and suppose that resources are sufficient to allow the players to use this equilibrium strategy in each trial. We now address the problem of whether the stochastic replicator dynamics converge to the state where all the members of the population use the Nash equilibrium in every trial. Corollary 4.1, below, provides a sufficient condition for almost sure convergence. The result will be applied to the sperm competition conflict of Subsection 3.3.

In general, it is not clear whether, in the above situation, an evolutionary process will converge to the state where everyone always uses the Nash equilibrium. In fact, consider the prisoner's dilemma game where, in every trial, players may defect or cooperate. 'Defect' is the unique strict Nash equilibrium in every trial, and 'always defect' is the unique strict Nash equilibrium in the repeated prisoner's dilemma game. Imhof et al. (2005) examined a frequency-dependent Moran process to understand the evolution of a finite population playing the repeated prisoner's dilemma game. It turned out that, depending on the population size and on the number of rounds, the population may most of the time be far from playing 'always defect'. For another example, consider the stochastic replicator dynamics describing an infinite population playing a one-trial prisoner's dilemma game. Depending on the sizes of the coefficients $\sigma_{j}$, these dynamics may converge almost surely to the state where everyone cooperates; see Fudenberg and Harris (1992, p. 428) and Imhof (2005, pp. 1029-1030).

We first prove a general convergence theorem with conditions given in terms of the payoff matrix $\boldsymbol{A}$. From this we derive a convergence result for multiple-trial conflicts under conditions on the one-trial payoff matrix $\boldsymbol{A}^{(1)}$.

Theorem 4.5. Let $\boldsymbol{X}(t)$ be given by (2.4). Suppose that strategy $k$ is a strict Nash equilibrium of the underlying payoff matrix $\boldsymbol{A}$ and that

$$
\max \left\{\sigma_{1}^{2}, \ldots, \sigma_{n}^{2}\right\}<\frac{2}{3} \min \left\{a_{k k}-a_{j k}: j \neq k\right\} .
$$

Suppose further that $\boldsymbol{A}$ satisfies the weak inequality

$$
\boldsymbol{z}^{\top} \boldsymbol{A} \boldsymbol{z} \leq 0 \quad \text { for all } z \in \mathcal{Z}
$$

where

$$
\mathcal{Z}=\left\{z \in \mathbb{R}^{n}: z \neq \mathbf{0}, z_{1}+\cdots+z_{n}=0, z_{j} \geq 0 \text { for all } j \neq k\right\} .
$$

Then, for every initial state $\boldsymbol{x} \in \operatorname{int}(\Delta)$,

$$
\mathrm{P}_{\boldsymbol{x}}\left\{\lim _{t \rightarrow \infty} \boldsymbol{X}(t)=\boldsymbol{e}_{k}\right\}=1 .
$$

Proof. Let $\phi(\boldsymbol{y})=-\log y_{k}$. Let $\mathcal{A}$ be given by (4.5). Then

$$
\mathcal{A} \phi(\boldsymbol{y})=\left(\boldsymbol{y}-\boldsymbol{e}_{k}\right)^{\top} \tilde{\boldsymbol{A}} \boldsymbol{y}+\frac{1}{2} \sum_{j=1}^{n} \sigma_{j}^{2}\left(y_{j}-y_{j}^{2}\right),
$$


where $\tilde{\boldsymbol{A}}=\left(\tilde{a}_{i j}\right)$ and $\tilde{a}_{i j}=a_{i j}-\frac{1}{2} \sigma_{i}^{2}$. For all $\boldsymbol{y} \in \Delta$,

$$
\frac{1}{2} \sum_{j=1}^{n} \sigma_{j}^{2}\left(y_{j}-y_{j}^{2}\right) \leq \frac{1}{2} \sigma_{k}^{2}\left(1-y_{k}\right)+\frac{1}{2} \sum_{j \neq k} \sigma_{j}^{2} y_{j} \leq \max \left\{\sigma_{1}^{2}, \ldots, \sigma_{n}^{2}\right\}\left(1-y_{k}\right) .
$$

If $z \in \mathcal{Z}$ then $\boldsymbol{A} z=\tilde{\boldsymbol{A}} \boldsymbol{z}$, and so, by (4.12), $\boldsymbol{z}^{\top} \tilde{\boldsymbol{A}} \boldsymbol{z} \leq 0$. Hence, for all $\boldsymbol{y} \in \Delta$,

$$
\left(\boldsymbol{y}-\boldsymbol{e}_{k}\right)^{\top} \tilde{\boldsymbol{A}} \boldsymbol{y} \leq\left(\boldsymbol{y}-\boldsymbol{e}_{k}\right)^{\top} \tilde{\boldsymbol{A}} \boldsymbol{e}_{k}=\left(y_{k}-1\right) \tilde{a}_{k k}+\sum_{j \neq k} y_{j} \tilde{a}_{j k} \leq\left(1-y_{k}\right)\left(-\tilde{a}_{k k}+\max _{j \neq k} \tilde{a}_{j k}\right) \text {. }
$$

By assumption (4.11), there exists $\gamma>0$ such that

$$
\begin{aligned}
-\tilde{a}_{k k}+\max _{j \neq k} \tilde{a}_{j k} & \leq-a_{k k}+\frac{1}{2} \sigma_{k}^{2}+\max _{j \neq k} a_{j k} \\
& \leq-\frac{3}{2} \max \left\{\sigma_{1}^{2}, \ldots, \sigma_{n}^{2}\right\}-\gamma+\frac{1}{2} \sigma_{k}^{2} \\
& \leq-\max \left\{\sigma_{1}^{2}, \ldots, \sigma_{n}^{2}\right\}-\gamma .
\end{aligned}
$$

It now follows that

$$
\mathcal{A} \phi(\boldsymbol{y}) \leq-\gamma\left(1-y_{k}\right) \text { for all } \boldsymbol{y} \in \operatorname{int}(\Delta) .
$$

Therefore, for every $\varepsilon>0$, there is some $\delta>0$ such that

$$
\mathrm{P}_{\boldsymbol{x}}\left\{X(t) \rightarrow \boldsymbol{e}_{k}\right\} \geq 1-\varepsilon \quad \text { for all } \boldsymbol{x} \in \operatorname{int}(\Delta) \text { with } x_{k} \geq 1-\delta ;
$$

see Gichman and Skorochod (1971, p. 314). Let $\eta:=\inf \left\{t \geq 0: X_{k}(t) \geq 1-\delta\right\}$. For $K>0$, let $\tau_{K}:=\inf \{t \geq 0: \phi(X(t)) \geq K\}$. By Dynkin's formula and (4.13), for every $t>0$ and every $x \in \operatorname{int}(\Delta)$,

$0 \leq \mathrm{E}_{\boldsymbol{x}} \phi\left(\boldsymbol{X}\left(t \wedge \tau_{K} \wedge \eta\right)\right)=\phi(\boldsymbol{x})+\mathrm{E}_{\boldsymbol{x}} \int_{0}^{t \wedge \tau_{K} \wedge \eta} \mathcal{A} \phi(\boldsymbol{X}(s)) \mathrm{d} s \leq \phi(\boldsymbol{x})-\gamma \delta \mathrm{E}_{\boldsymbol{x}}\left(t \wedge \tau_{K} \wedge \eta\right)$.

Letting $t \nearrow \infty$ and $K \nearrow \infty$, we obtain, by monotone convergence, $\mathrm{E}_{\boldsymbol{x}} \eta \leq \phi(\boldsymbol{x}) /(\gamma \delta)<\infty$, so that $\eta$ is $\mathrm{P}_{\boldsymbol{x}}$-almost surely finite. Set $F=\left\{\boldsymbol{X}(t) \rightarrow \boldsymbol{e}_{k}\right\}$, and let $\mathbf{1}_{F}$ denote the indicator function of $F$. In view of the strong Markov property of $\{X(t)\}$, it follows from (4.14) that

$$
\mathrm{P}_{\boldsymbol{x}}\left\{\boldsymbol{X}(t) \rightarrow \boldsymbol{e}_{k}\right\}=\mathrm{E}_{\boldsymbol{x}} \mathbf{1}_{F}=\mathrm{E}_{\boldsymbol{x}} \mathrm{E}_{\boldsymbol{X}(\eta)} \mathbf{1}_{F} \geq 1-\varepsilon .
$$

But $\varepsilon>0$ was arbitrary, and the proof is complete.

Remark 4.3. Condition (4.11) implies that $\boldsymbol{e}_{k}$ is stochastically stable, that is, for every open neighbourhood $U$ of $\boldsymbol{e}_{k}$,

$$
\lim _{\substack{x \rightarrow e_{k} \\ x \in \operatorname{int}(\Delta)}} \mathrm{P}_{\boldsymbol{x}}\{X(t) \in U \text { for all } t \geq 0\}=1 ;
$$

see Theorem 4.1 of Imhof (2005). Thus, under the assumptions of Theorem 4.5, $\boldsymbol{e}_{k}$ is (stochastically asymptotically) stable in the large (Has'minskir (1980, p. 169)). Neither (4.11) nor (4.12) is necessary for stability in the large. For example, if $n=2, k=1, a_{11}=2, a_{12}=1$, and $a_{21}=a_{22}=0$, then, by Proposition 1(i) of Fudenberg and Harris (1992) and Theorem 4.11(a) of Hofbauer and Imhof (2007), $\boldsymbol{e}_{1}$ is stable in the large whenever $\sigma_{1}=\sigma_{2}>0$. However, (4.12) does not hold and (4.11) is violated if $\sigma_{1}^{2}=\sigma_{2}^{2} \geq \frac{4}{3}$. A necessary condition for the weaker conclusion that $\mathrm{P}_{\boldsymbol{x}}\left\{\boldsymbol{X}(t) \rightarrow \boldsymbol{e}_{k}\right\}>0$ for some $\boldsymbol{x} \in \operatorname{int}(\Delta)$ is that $a_{k k}-a_{j k} \geq \frac{1}{2}\left(\sigma_{k}^{2}-\sigma_{j}^{2}\right)$ for all $j \neq k$; see Theorem 4.11(b) of Hofbauer and Imhof (2007). 
Consider the general model for multiple-trial conflicts of Subsection 3.1. Again, denote the pure strategies in $\mathbb{U}$ by $1, \ldots, n$. Thus, the indices $1, \ldots, n$ of the components of vectors in $\Delta \subset \mathbb{R}^{n}$ correspond to the elements of $\mathbb{U}$ in a fixed order. This yields a one-to-one relation between the points in the state space $\Delta$ and the mixed strategies in $\mathcal{P}$. In particular, the $n$ vertices of $\Delta$ correspond to the pure strategies in the $m$-trial conflict.

Corollary 4.1. Let $\boldsymbol{X}(t)$ be given by the stochastic replicator dynamics (2.4) for the $m$-trial model of Subsection 3.1. Suppose that the one-trial payoff matrix $\boldsymbol{A}^{(1)}$ is conditionally negative semidefinite and that $k$ is a strict Nash equilibrium in each trial. Suppose also that $(k, \ldots, k) \in \mathbb{U}$, and let $\boldsymbol{e}$ denote the corresponding vertex of $\Delta$. Then, for every initial state $\boldsymbol{x} \in \operatorname{int}(\Delta), \mathrm{P}_{\boldsymbol{x}}\{\boldsymbol{X}(t) \rightarrow \boldsymbol{e}\}=1$, provided that $\sigma_{1}, \ldots, \sigma_{n}$ are sufficiently small.

Proof. The assumption that $\boldsymbol{A}^{(1)}$ is conditionally negative semidefinite implies that the multiple-trial payoff matrix $\boldsymbol{A}$ is conditionally negative semidefinite as well. This can be verified using (3.1). Condition (4.12) is therefore met. Moreover, by Theorem 3.2, $\boldsymbol{e}$ is a strict Nash equilibrium for $\boldsymbol{A}$. In particular, condition (4.11) is satisfied when $\sigma_{1}, \ldots, \sigma_{n}$ are small enough. The assertion follows from Theorem 4.5.

Example 4.1. Consider the repeated prisoner's dilemma game, where in each trial the pure strategies are $1=$ 'cooperate' and $2=$ 'defect', and the one-trial payoff matrix is

$$
\boldsymbol{A}^{(1)}=\left(\begin{array}{ll}
3 & 0 \\
5 & 1
\end{array}\right) \text {. }
$$

The matrix $\boldsymbol{A}^{(1)}$ is conditionally negative definite. Suppose that players do not incur any costs, so that they can choose freely between cooperation and defection. It follows from Corollary 4.1 that the stochastic replicator dynamics converge almost surely to the state where everyone defects in every trial, provided that the coefficients $\sigma_{j}$ are sufficiently small. This result is not at variance with the fact that, in the stochastic model of Imhof et al. (2005), the population may show cooperative behaviour most of the time. Indeed, that this type of behaviour occurs has been proved only for finite populations of moderate size, while the present replicator model describes infinite populations. It can be shown that if in the finite population model the size of the population tends to $\infty$, noncooperative behaviour becomes prevalent.

We now return to the multiple-trial sperm competition game. Corollary 4.1 and Theorem 3.4 yield at once the following result on the asymptotic behaviour of the stochastic dynamics.

Theorem 4.6. Let $\boldsymbol{X}(t)$ be given by the stochastic replicator dynamics (2.4) for the $m$-trial sperm competition game with fair raffles described in Subsection 3.3. Let $k$ be given by (3.9), and suppose that $m c_{k} \leq L$. Let $\boldsymbol{e}$ be the vertex of $\Delta$ that corresponds to the pure $m$-trial strategy $(k, \ldots, k)$. Then, for every initial state $\boldsymbol{x} \in \operatorname{int}(\Delta), \mathrm{P}_{\boldsymbol{x}}\{\boldsymbol{X}(t) \rightarrow \boldsymbol{e}\}=1$, provided that $\sigma_{1}, \ldots, \sigma_{n}$ are sufficiently small.

Thus, in the sperm competition game, there is a strategy that represents an optimal compromise between cost and benefit, and if the males are able to use that strategy in every round, the population evolves towards a state where the males implement the optimal compromise in every round. A similar result for various deterministic dynamics has been shown by Fryer et al. (1999) for two-trial sperm competition games which accommodate the possibility that males fail to obtain matings. 


\subsection{Weakly dominated strategies}

In a game with payoff matrix $\boldsymbol{A}$, the pure strategy $k$ is said to be weakly dominated by strategy $p \in \Delta$ if

$$
\boldsymbol{e}_{k}^{\top} \boldsymbol{A} \boldsymbol{q} \leq \boldsymbol{p}^{\top} \boldsymbol{A q} \text { for all } \boldsymbol{q} \in \Delta
$$

with strict inequality for some $\boldsymbol{q} \in \Delta$. If the inequality is strict for all $\boldsymbol{q} \in \Delta$ then $k$ is said to be strictly dominated by $\boldsymbol{p}$.

For instance, in the two-trial war of attrition of Subsection 3.2 with $V=L=2$, strategy $(0,0)$ is weakly but not strictly dominated by the mixed strategy that assigns probability $\frac{1}{2}$ to $(0,1)$ and $(2,0)$.

Under the deterministic replicator dynamics, (2.2), the frequency of $k$-players grows if and only if their current payoff is larger than the average payoff of the population. Even if $k$ is strictly dominated, the payoff to $k$ may be above the current average. It is therefore not at all obvious whether dominated strategies will become extinct, that is, whether their frequencies converge to 0. Akin (1980) has shown that strictly dominated pure strategies do become extinct, and Samuelson and Zhang (1992) have proved that even every iteratively dominated pure strategy vanishes in the long run. It has been shown by Cabrales (2000) that these results continue to hold for the stochastic replicator dynamics, provided that the diffusion coefficients are small enough. If $\sigma_{1}=\cdots=\sigma_{n}$ then strictly dominated strategies die out exponentially quickly; see Imhof (2005).

The deterministic and the stochastic replicator dynamics differ when weakly dominated strategies are considered: under the deterministic dynamics, the frequency of a weakly dominated strategy may stay above a fixed positive number, but it will be shown in Theorem 4.7, below, that this does not happen under the stochastic dynamics. On the other hand, in Example 4.2, below, a weakly dominated strategy dies out in the deterministic model but persists in the stochastic model.

Note that if $k$ is weakly dominated by a mixed strategy $p \in \Delta$ then there must exist some pure strategy $l$ such that $\boldsymbol{e}_{k}^{\top} \boldsymbol{A} \boldsymbol{e}_{l}<\boldsymbol{p}^{\top} \boldsymbol{A} \boldsymbol{e}_{l}$.

Theorem 4.7. Let $\boldsymbol{X}(t)$ be given by (2.4) with initial state $\boldsymbol{X}(0)=\boldsymbol{x} \in \operatorname{int}(\Delta)$, and let $\sigma_{1}=\sigma_{2}=\cdots=\sigma_{n}>0$. Suppose that the pure strategy $k$ is weakly dominated by some strategy $\boldsymbol{p} \in \Delta$. Then

$$
\mathrm{P}_{\boldsymbol{x}}\left\{\liminf _{t \rightarrow \infty} X_{k}(t)=0\right\}=1 .
$$

Moreover, if l is one of the pure strategies for which $\boldsymbol{e}_{k}^{\top} \boldsymbol{A} \boldsymbol{e}_{l}<\boldsymbol{p}^{\top} \boldsymbol{A} \boldsymbol{e}_{l}$ then

$$
\mathrm{P}_{\boldsymbol{x}}\left\{\lim _{t \rightarrow \infty} X_{k}(t)=0 \text { or } \liminf _{t \rightarrow \infty} X_{l}(t)=0\right\}=1 .
$$

Proof. Let $Z_{1}(t), \ldots, Z_{n}(t)$ be given by (2.3). Set

$$
Q(t)=\log Z_{k}(t)-\sum_{j=1}^{n} p_{j} \log Z_{j}(t)=\log X_{k}(t)-\sum_{j=1}^{n} p_{j} \log X_{j}(t), \quad t \geq 0 .
$$

We have

$$
\mathrm{d} Q(t)=\left(\boldsymbol{e}_{k}^{\top} \boldsymbol{A} \boldsymbol{X}(s)-\boldsymbol{p}^{\top} \boldsymbol{A} \boldsymbol{X}(s)-\frac{\sigma_{k}^{2}}{2}+\frac{1}{2} \sum_{j=1}^{n} p_{j} \sigma_{j}^{2}\right) \mathrm{d} t+\sigma_{k} \mathrm{~d} W_{k}(t)-\sum_{j=1}^{n} p_{j} \sigma_{j} \mathrm{~d} W_{j}(t) .
$$


As $k$ is weakly dominated by $\boldsymbol{p}, \boldsymbol{e}_{k}^{\top} \boldsymbol{A} \boldsymbol{X}(s)-\boldsymbol{p}^{\top} \boldsymbol{A} \boldsymbol{X}(s) \leq 0$. Furthermore, $\sum_{j=1}^{n} p_{j} \sigma_{j}^{2}=\sigma_{k}^{2}$. Hence,

$$
\log X_{k}(t) \leq Q(t) \leq Q(0)+\sigma_{1}\left(W_{k}(t)-\sum_{j=1}^{n} p_{j} W_{j}(t)\right)=Q(0)+\tilde{\sigma} \tilde{W}(t),
$$

where $\tilde{\sigma}=\sigma_{1}\left(\left(1-p_{k}\right)^{2}+\sum_{j \neq k} p_{j}^{2}\right)^{1 / 2}>0$ and $\tilde{W}(t)$ is a standard Brownian motion. This proves the first assertion since, with probability $1, \liminf _{t \rightarrow \infty} \tilde{W}(t)=-\infty$.

To prove the second assertion, let $l$ be such that $\varepsilon_{1}:=\boldsymbol{p}^{\top} \boldsymbol{A} \boldsymbol{e}_{l}-\boldsymbol{e}_{k}^{\top} \boldsymbol{A} \boldsymbol{e}_{l}>0$. For every $\boldsymbol{y} \in \operatorname{int}(\Delta)$, let $\boldsymbol{z}(\boldsymbol{y}):=\left(1-y_{l}\right)^{-1}\left(\boldsymbol{y}-y_{l} \boldsymbol{e}_{l}\right)$. Clearly, $\boldsymbol{z}(\boldsymbol{y}) \in \Delta$, and so

$$
\begin{aligned}
\left(\boldsymbol{e}_{k}-\boldsymbol{p}\right)^{\top} \boldsymbol{A} \boldsymbol{y} & =\left(1-y_{l}\right)\left(\boldsymbol{e}_{k}-\boldsymbol{p}\right)^{\top} \boldsymbol{A} \boldsymbol{z}(\boldsymbol{y})+y_{l}\left(\boldsymbol{e}_{k}-\boldsymbol{p}\right)^{\top} \boldsymbol{A} \boldsymbol{e}_{l} \\
& \leq y_{l}\left(\boldsymbol{e}_{k}-\boldsymbol{p}\right)^{\top} \boldsymbol{A} \boldsymbol{e}_{l} \\
& =-\varepsilon_{1} y_{l} .
\end{aligned}
$$

By the law of the iterated logarithm for Brownian motion, there is a null set $\Omega_{0}$ such that

$$
\limsup _{t \rightarrow \infty} \frac{\tilde{W}(t, \omega)}{\sqrt{2 t \log \log t}}=1 \quad \text { for all } \omega \in \Omega_{0}^{C} .
$$

Now suppose that $\omega \in \Omega_{0}^{C}$ is such that $\varepsilon_{2}:=\liminf _{t \rightarrow \infty} X_{l}(t, \omega)>0$. Then there exists $T(\omega)>0$ such that

$$
X_{l}(t, \omega)>\frac{\varepsilon_{2}}{2} \quad \text { and } \quad \tilde{W}(t, \omega) \leq t^{3 / 4} \quad \text { for all } t \geq T(\omega) .
$$

It follows from (4.15) and (4.16) that

$$
\begin{aligned}
\log X_{k}(t, \omega) & \leq Q(t, \omega) \\
& =Q(0, \omega)+\int_{0}^{t}\left(\boldsymbol{e}_{k}-\boldsymbol{p}\right)^{\top} \boldsymbol{A} \boldsymbol{X}(s, \omega) \mathrm{d} s+\tilde{\sigma} \tilde{W}(t, \omega) \\
& \leq Q(0, \omega)-\varepsilon_{1} \int_{0}^{t} X_{l}(s, \omega) \mathrm{d} s+\tilde{\sigma} \tilde{W}(t, \omega) \\
& \leq Q(0, \omega)-\frac{\varepsilon_{1} \varepsilon_{2}}{2}(t-T(\omega))+\tilde{\sigma} t^{3 / 4} \quad \text { for all } t \geq T(\omega)
\end{aligned}
$$

Thus, $\lim _{t \rightarrow \infty} X_{k}(t, \omega)=0$.

Example 4.2. Under the assumptions of Theorem 4.7, it is not in general true that $\mathrm{P}_{\boldsymbol{x}}\left\{X_{k}(t) \rightarrow\right.$ $0\}>0$. If

$$
\boldsymbol{A}=\left(\begin{array}{ll}
0 & 1 \\
1 & 1
\end{array}\right)
$$

then strategy 1 is weakly dominated by strategy 2 . It can be shown that $X(t)$ is recurrent (Friedman (1975, Theorem 7.1, p. 219)). In particular, $\mathrm{P}_{\boldsymbol{x}}\left\{X_{1}(t) \rightarrow 0\right\}=0$ for every $\boldsymbol{x} \in$ $\operatorname{int}(\Delta)$. On the other hand, for the deterministic dynamics, $(2.2), \xi_{1}(t) \rightarrow 0$ for every initial condition $\xi(0)=x \in \operatorname{int}(\Delta)$. 


\section{Appendix A. Proof of Theorem 3.3}

The proofs of (a) and (b) are similar and so only that of (a) will be given. Thus, let $L=2 K+1$, and assume that $V$ satisfies (3.3). Define $p$ by (3.4) and (3.5). Write $\vartheta=1+V^{-1}$ and $u_{k}=U_{k}(\vartheta)$. As $\vartheta>1, u_{-1}<u_{0}<u_{1}<\cdots$, which implies that $p(s) \geq 0$ for all $s \in \mathbb{U}$. A telescope argument shows that $\sum_{s \in \mathbb{U}} p(s)=1$, so that $p$ is indeed a probability measure on U.

Set

$$
\left(\begin{array}{c}
\gamma_{0} \\
\vdots \\
\gamma_{L}
\end{array}\right):=\boldsymbol{A}^{(1)} \boldsymbol{f}_{1}(p)=\boldsymbol{A}^{(1)} \boldsymbol{f}_{2}(p) .
$$

It will be shown that there exists a constant $c \in \mathbb{R}$ such that

$$
\gamma_{j}+\gamma_{L-j}=c, \quad j=0, \ldots, K
$$

and

$$
\gamma_{0}<\gamma_{1}<\cdots<\gamma_{L}
$$

Suppose for the moment that (A.1) and (A.2) are true. Then, for every $q \in \mathcal{P}$, by (3.1),

$$
\begin{aligned}
M(q, p) & =\left[\boldsymbol{f}_{1}(q)+\boldsymbol{f}_{2}(q)\right]^{\top}\left(\gamma_{0}, \ldots, \gamma_{L}\right)^{\top} \\
& =\sum_{\boldsymbol{s} \in \mathbb{U}} q(\boldsymbol{s})\left(\gamma_{s_{1}}+\gamma_{s_{2}}\right) \\
& \leq \sum_{\boldsymbol{s} \in \mathbb{U}} q(\boldsymbol{s})\left(\gamma_{s_{1}}+\gamma_{L-s_{1}}\right) \\
& =c \\
& =M(p, p) .
\end{aligned}
$$

That is, $p$ is a Nash equilibrium. As $\boldsymbol{A}^{(1)}$ is conditionally negative definite (Whittaker and Cannings (1994, proof of Theorem 5.1.1)), it follows from Theorem 3.1 that $p$ belongs to the unique ES set. It also follows that if $q$ is any member of the ES set then $f_{1}(q)=f_{2}(q)=f_{1}(p)$. Thus,

$$
f_{k, 1}(q)=p(k, L-k)=f_{L-k, 2}(q), \quad k=0, \ldots, L .
$$

It will now be shown by induction that this implies that, for $k=0, \ldots, L$,

$$
q(k, L-k)=p(k, L-k) \quad \text { and } \quad q(k, j)=0 \quad \text { for all } j \text { with } 0 \leq j<L-k .
$$

By (A.3), $q(0, L)=f_{L, 2}(q)=p(0, L)$ and

$$
\sum_{j=0}^{L} q(0, j)=f_{0,1}(q)=p(0, L),
$$

proving (A.4) for $k=0$. Let $\kappa \in\{1, \ldots, L\}$, and assume that (A.4) holds for $k=0, \ldots, \kappa-1$. Then, by (A.3),

$$
q(\kappa, L-\kappa)=\sum_{k=0}^{\kappa} q(k, L-\kappa)=f_{L-\kappa, 2}(q)=p(\kappa, L-\kappa)=f_{\kappa, 1}(q)=\sum_{j=0}^{L-\kappa} q(\kappa, j),
$$


proving (A.4) for $k=\kappa$. Thus, (A.4) holds for every $k$, which means that $q=p$. The ES set therefore contains only the element $p$, so that $p$ is an ES strategy.

Now, to prove (A.1), let $j \in\{0, \ldots, K\}$. Then

$$
\begin{aligned}
\gamma_{j}+\gamma_{L-j}= & \sum_{k=0}^{L}\left(a_{j k}^{(1)}+a_{L-j, k}^{(1)}\right) f_{k, 1}(p) \\
= & \sum_{k<j}(V-k) f_{k, 1}(p)+\sum_{k \geq j}(-j) f_{k, 1}(p) \\
& +\sum_{k<L-j}(V-k) f_{k, 1}(p)+\sum_{k \geq L-j}-(L-j) f_{k, 1}(p) \\
= & : I_{1}(j)+I_{2}(j)+I_{3}(j)+I_{4}(j) .
\end{aligned}
$$

To calculate $I_{1}(j), \ldots, I_{4}(j)$, note first that

$$
\begin{aligned}
& f_{k, 1}(p)=p(k, L-k)=\frac{u_{K-k}-u_{K-k-1}}{2 u_{K}}, \quad k=0, \ldots, K, \\
& f_{k, 1}(p)=\frac{u_{k-K-1}-u_{k-K-2}}{2 u_{K}}, \quad k=K+1, \ldots, L .
\end{aligned}
$$

Hence,

$$
\begin{aligned}
I_{1}(j) & =\frac{1}{2 u_{K}} \sum_{k=0}^{j-1}(V-k)\left(u_{K-k}-u_{K-k-1}\right) \\
& =\frac{1}{2 u_{K}}\left(\sum_{k=0}^{j-1}(V-k) u_{K-k}-\sum_{k=1}^{j}(V-k) u_{K-k}-\sum_{k=1}^{j} u_{K-k}\right) \\
& =\frac{1}{2 u_{K}}\left(V u_{K}-(V-j) u_{K-j}-\sum_{k=K-j}^{K-1} u_{k}\right) .
\end{aligned}
$$

Adding formulae (22.12.4) and (22.12.5) of Abramowitz and Stegun (1965, p. 785), we obtain

$$
\sum_{k=0}^{l} u_{k}=\sum_{k=0}^{l} U_{k}(\vartheta)=\frac{T_{l+1}(\vartheta)+T_{l+2}(\vartheta)-1-\vartheta}{2\left(\vartheta^{2}-1\right)} \quad \text { for } l \geq 0
$$

Hence,

$$
I_{1}(j)=\frac{1}{2 u_{K}}\left(V u_{K}-(V-j) u_{K-j}-\frac{T_{K}(\vartheta)+T_{K+1}(\vartheta)-T_{K-j}(\vartheta)-T_{K-j+1}(\vartheta)}{2\left(\vartheta^{2}-1\right)}\right) .
$$

Also,

$$
\begin{aligned}
I_{2}(j) & =\frac{-j}{2 u_{K}}\left(\sum_{k=j}^{K}\left(u_{K-k}-u_{K-k-1}\right)+\sum_{k=K+1}^{2 K+1}\left(u_{k-K-1}-u_{k-K-2}\right)\right) \\
& =\frac{-j u_{K-j}}{2 u_{K}}-\frac{j}{2},
\end{aligned}
$$




$$
\begin{aligned}
& I_{3}(j)=I_{1}(j)+\frac{1}{2 u_{K}}\left(\sum_{k=j}^{K}(V-k)\left(u_{K-k}-u_{K-k-1}\right)\right. \\
&\left.\quad+\sum_{k=K+1}^{2 K-j}(V-k)\left(u_{k-K-1}-u_{k-K-2}\right)\right) \\
&=I_{1}(j)+\frac{1}{2 u_{K}}\left((V-j)\left(u_{K-j}-u_{K-j-1}\right)\right.\left.\quad+(2 V-2 K-1) \sum_{k=j+1}^{K}\left(u_{K-k}-u_{K-k-1}\right)\right) \\
&=I_{1}(j)+\frac{1}{2 u_{K}}\left((V-j) u_{K-j}+(V-2 K+j-1) u_{K-j-1}\right),
\end{aligned}
$$

and

$$
I_{4}(j)=\frac{j-L}{2 u_{K}} \sum_{k=2 K+1-j}^{2 K+1}\left(u_{k-K-1}-u_{k-K-2}\right)=(j-L)\left(\frac{1}{2}-\frac{u_{K-j-1}}{2 u_{k}}\right) .
$$

It now follows that, for $j=0, \ldots, K$,

$$
\begin{aligned}
\gamma_{j}+\gamma_{L-j}= & I_{1}(j)+I_{2}(j)+I_{3}(j)+I_{4}(j) \\
= & V-\frac{L}{2}+\frac{u_{K-j-1}-u_{K-j}}{2 u_{K}} V \\
& +\frac{T_{K-j}(\vartheta)+T_{K-j+1}(\vartheta)-T_{K}(\vartheta)-T_{K+1}(\vartheta)}{2 u_{K}\left(\vartheta^{2}-1\right)} .
\end{aligned}
$$

By formulae (22.5.6) and (22.5.7) of Abramowitz and Stegun (1965, pp. 777-778),

$$
T_{K-j}(\vartheta)+T_{K-j+1}(\vartheta)=(1+\vartheta)\left(U_{K-j}(\vartheta)-U_{K-j-1}(\vartheta)\right),
$$

and so, since $\vartheta-1=V^{-1}$,

$$
\gamma_{j}+\gamma_{L-j}=V-\frac{L}{2}-\frac{T_{K}(\vartheta)+T_{K+1}(\vartheta)}{2 u_{K}\left(\vartheta^{2}-1\right)}=: c,
$$

which is independent of $j$. This proves (A.1).

It remains to prove (A.2). For $j \in\{0, \ldots, K\}$,

$$
\begin{aligned}
\gamma_{j+1}-\gamma_{j} & =\sum_{k=0}^{L}\left(a_{j+1, k}^{(1)}-a_{j k}^{(1)}\right) f_{k, 1}(p) \\
& =V f_{j, 1}(p)-\sum_{k=j+1}^{L} f_{k, 1}(p) \\
& =V \frac{u_{K-j}-u_{K-j-1}}{2 u_{K}}-\frac{1}{2}-\frac{u_{K-j-1}}{2 u_{K}} .
\end{aligned}
$$


Again using (22.5.6) of Abramowitz and Stegun (1965) and assumption (3.3) on $V$, we obtain

$$
\gamma_{j+1}-\gamma_{j}=V \frac{T_{K-j}(\vartheta)}{2 u_{K}}-\frac{1}{2} \geq \frac{V}{2 u_{K}}-\frac{1}{2}>0 .
$$

That $\gamma_{j+1}>\gamma_{j}$ for $j=K+1, \ldots, L-1$, too, is now a consequence of (A.1).

\section{Appendix B. Proof of Theorem 3.4}

It will first be shown that the matrix $\boldsymbol{A}^{(1)}$ given by (3.7) is conditionally negative semidefinite. If $z=\left(z_{0}, \ldots, z_{v}\right)^{\top}$ is such that $z_{0}+\cdots+z_{v}=0$ then (see Haigh (1975))

$$
\boldsymbol{z}^{\top} \boldsymbol{A}^{(1)} \boldsymbol{z}=\sum_{i, j=0}^{\nu-1} z_{i} b_{i j} z_{j}
$$

where

$$
\begin{aligned}
b_{i j} & =a_{i j}^{(1)}+a_{v v}^{(1)}-a_{i v}^{(1)}-a_{v j}^{(1)} \\
& =\left(\frac{\eta_{i} \phi\left(\eta_{i}+\eta_{j}\right)}{\eta_{i}+\eta_{j}}+\frac{\phi\left(2 \eta_{v}\right)}{2}-\frac{\eta_{i} \phi\left(\eta_{i}+\eta_{v}\right)}{\eta_{i}+\eta_{v}}-\frac{\eta_{\nu} \phi\left(\eta_{j}+\eta_{v}\right)}{\eta_{j}+\eta_{v}}\right) V \\
& =\left(\frac{\eta_{i}}{\eta_{i}+\eta_{j}}+\frac{1}{2}-\frac{\eta_{i}}{\eta_{i}+\eta_{\nu}}-\frac{\eta_{v}}{\eta_{j}+\eta_{v}}\right) \alpha V .
\end{aligned}
$$

Hence, $b_{i j}+b_{j i}=0$, and so

$$
\boldsymbol{z}^{\top} \boldsymbol{A}^{(1)} \boldsymbol{z}=\frac{1}{2} \sum_{i, j=0}^{\nu-1} z_{i}\left(b_{i j}+b_{j i}\right) z_{j}=0
$$

Now suppose that $V \in(0, \infty) \backslash\left\{\rho_{0}, \ldots, \rho_{v-1}\right\}$, and let $k$ be given by (3.9). If $k \leq v-1$ then

$$
a_{k k}^{(1)}-a_{k+1, k}^{(1)}=c_{k+1}-c_{k}+\left(\frac{\phi\left(2 \eta_{k}\right)}{2}-\frac{\eta_{k+1} \phi\left(\eta_{k}+\eta_{k+1}\right)}{\eta_{k}+\eta_{k+1}}\right) V .
$$

Since

$$
\frac{\phi\left(2 \eta_{k}\right)}{2}-\frac{\eta_{k+1} \phi\left(\eta_{k}+\eta_{k+1}\right)}{\eta_{k}+\eta_{k+1}}=\frac{\eta_{k}-\eta_{k+1}}{2}\left(\frac{\phi\left(\eta_{k}+\eta_{k+1}\right)}{\eta_{k}+\eta_{k+1}}+\beta\right)<0
$$

and $V<\rho_{k}$, it follows that

$$
a_{k k}^{(1)}>a_{k+1, k}^{(1)}+c_{k+1}-c_{k}+\frac{\eta_{k}-\eta_{k+1}}{2}\left(\frac{\phi\left(\eta_{k}+\eta_{k+1}\right)}{\eta_{k}+\eta_{k+1}}+\beta\right) \rho_{k}=a_{k+1, k}^{(1)},
$$

provided that $k \leq v-1$. A similar calculation shows that

$$
a_{k k}^{(1)}>a_{k-1, k}^{(1)}
$$

provided that $k \geq 1$. Let

$$
g_{1}(x)=\frac{x \phi\left(x+\eta_{k}\right)}{x+\eta_{k}}, \quad \eta_{0} \leq x \leq \eta_{\nu} .
$$


Then $g_{1}^{\prime \prime}(x)=-2 \alpha \eta_{k} /\left(x+\eta_{k}\right)^{3}$, so that $g_{1}$ is concave. Define a piecewise linear spline $g_{2}(x)$ on the interval $\left[\eta_{0}, \eta_{\nu}\right]$ by

$$
g_{2}(x)=\frac{x-\eta_{j}}{\eta_{j+1}-\eta_{j}} c_{j+1}+\frac{\eta_{j+1}-x}{\eta_{j+1}-\eta_{j}} c_{j} \quad \text { for } x \in\left[\eta_{j}, \eta_{j+1}\right], j \in\{0, \ldots, v-1\} .
$$

Condition (3.6) ensures that $g_{2}$ is convex. Thus, $g(x)=g_{1}(x) V-g_{2}(x)$ is concave, and $g\left(\eta_{j}\right)=a_{j k}^{(1)}$ for all $j$. In view of (B.2) and (B.3), it follows that

$$
a_{j k}^{(1)}<a_{k k}^{(1)} \quad \text { for all } j \neq k .
$$

That is, strategy $k$ is a strict Nash equilibrium for the one-trial game, and Theorem 3.2 yields the assertion in the present case.

Next suppose that $V=\rho_{k}$. Then

$$
a_{k k}^{(1)}=a_{k+1, k}^{(1)}=: \gamma, \quad a_{k, k+1}^{(1)}=a_{k+1, k+1}^{(1)}=: \delta,
$$

say. It follows as above that

$$
a_{j k}^{(1)}<\gamma \quad \text { and } \quad a_{j, k+1}^{(1)}<\delta \quad \text { for all } j \neq k, k+1 .
$$

Let $\mathcal{Q} \subset \mathcal{P}$ denote the set of all mixed strategies whose support is contained in $\{k, k+1\}^{m}$. Thus, $p \in \mathcal{Q}$ if and only if $f_{j, \mu}(p)=0$ for all $j \in\{0, \ldots, v\} \backslash\{k, k+1\}$ and all $\mu=1, \ldots, m$. If $q \in Q$ then, by (3.1), for all $p \in \mathcal{P}$,

$$
M(p, q)=\sum_{\mu=1}^{m} \boldsymbol{f}_{\mu}(p)^{\top} \boldsymbol{A}^{(1)} \boldsymbol{f}_{\mu}(q)=\sum_{\mu=1}^{m} \sum_{i=0}^{\nu} \sum_{j=k}^{k+1} f_{i, \mu}(p) a_{i j}^{(1)} f_{j, \mu}(q) .
$$

Moreover, if $q \in \mathcal{Q}$ and $p \in \mathcal{Q}$ then, by (B.4),

$$
M(p, q)=\sum_{\mu=1}^{m} \sum_{i=k}^{k+1} \sum_{j=k}^{k+1} f_{i, \mu}(p) a_{i j}^{(1)} f_{j, \mu}(q)=\sum_{\mu=1}^{m} \gamma f_{k, \mu}(q)+\delta f_{k+1, \mu}(q) .
$$

This shows that every pair of strategies in $\mathcal{Q}$ is an evolutionarily neutral pair. If $q \in \mathcal{Q}$ and $p \in \mathcal{P} \backslash \mathcal{Q}$ then $f_{i, \mu}(p)>0$ for some $\mu$ and some $i \neq k, k+1$. Hence, by (B.5),

$$
M(p, q)<\sum_{\mu=1}^{m} \gamma f_{k, \mu}(q)+\delta f_{k+1, \mu}(q)=M(q, q) .
$$

Thus, $\mathcal{Q}$ is an ES set. Finally, if $q \in \mathcal{Q}$ and $p \in \mathcal{P} \backslash \mathcal{Q}$ then

$$
\begin{aligned}
M(p, p)-M(q, p) & <M(p, p)-M(q, p)+M(q, q)-M(p, q) \\
& =\sum_{i=1}^{n}\left(f_{i}(p)-f_{i}(q)\right)^{\top} \boldsymbol{A}^{(1)}\left(f_{i}(p)-\boldsymbol{f}(q)\right) \\
& =0,
\end{aligned}
$$

by (B.1). It follows that there cannot be another ES set. 


\section{Acknowledgements}

I am grateful to W. H. Sandholm and the anonymous referee for several very helpful suggestions.

\section{References}

Abramowitz, M. and Stegun, I. A. (1965). Handbook of Mathematical Functions. Dover, New York.

AKIN, E. (1980). Domination or equilibrium. Math. Biosci. 50, 239-250.

Benaïm, M., Hofbauer, J. And Sandholm, W. H. (2008). Robust permanence and impermanence for stochastic replicator dynamics. J. Biol. Dyn. 2, 180-195.

Benaïm, M., Schreiber, S. J. And Tarrès, P. (2004). Generalized urn models of evolutionary processes. Ann. Appl. Prob. 14, 1455-1478.

Bishop, D. T. And Cannings, C. (1978). A generalized war of attrition. J. Theoret. Biol. 70, 85-124.

Cabrales, A. (2000). Stochastic replicator dynamics. Internat. Econom. Rev. 41, 451-481.

Cannings, C. and Whittaker, J. C. (1995). The finite horizon war of attrition. Games Econom. Behav. 11, $193-236$.

Cressman, R. (1992). The Stability Concept of Evolutionary Game Theory. Springer, Berlin.

Cressman, R. (2003). Evolutionary Dynamics and Extensive Form Games. MIT Press, Cambridge, MA.

Foster, D. And Young, P. (1990). Stochastic evolutionary game dynamics. Theoret. Pop. Biol. 38, 219-232. (Correction 51 (1997), 77-78.)

Friedman, A. (1975). Stochastic Differential Equations and Applications, Vol. 1. Academic Press, New York.

Fryer, T., Cannings, C. ANd Vickers, G. T. (1999). Sperm competition. I. Basic model, ESS and dynamics. J. Theoret. Biol. 196, 81-100.

FUdENBERG, D. AND HARRIS, C. (1992). Evolutionary dynamics with aggregate shocks. J. Econom. Theory 57, 420-441.

Gichman, I. I. AND Skorochod, A. W. (1971). Stochastische Differentialgleichungen. Akademie, Berlin.

Haigh, J. (1975). Game theory and evolution. Adv. Appl. Prob. 7, 8-11.

Has'minskĭ̌, R. Z. (1980). Stochastic Stability of Differential Equations. Sijthoff and Noordhoff, Germantown, MD.

Hofbauer, J. AND ImHof, L. A. (2007). Time averages, recurrence and transience in the stochastic replicator dynamics. Preprint, Bonn University.

Hofbauer, J. And Sigmund, K. (1998). Evolutionary Games and Population Dynamics. Cambridge University Press.

Hofbauer, J. And Sigmund, K. (2003). Evolutionary game dynamics. Bull. Amer. Math. Soc. (N.S.) 40, 479-519.

ImHof, L. A. (2005). The long-run behavior of the stochastic replicator dynamics. Ann. Appl. Prob. 15, 1019-1045.

Imhof, L. A., Fudenberg, D. AND Nowak, M. A. (2005). Evolutionary cycles of cooperation and defection. Proc. Nat. Acad. Sci. USA 102, 10797-10800.

Karlin, S. (1959). Mathematical Methods and Theory in Games, Programming, and Economics, Vol. 1. AddisonWesley, Reading, MA.

Karlin, S. (1968). Total Positivity. Stanford University Press.

KhasminskiI, R. AND Potsepun, N. (2006). On the replicator dynamics behavior under Stratonovich type random perturbations. Stoch. Dyn. 6, 197-211.

Maynard Smith, J. and Price, G. R. (1973). The logic of animal conflict. Nature 246, 15-18.

NowaK, M. A. AND Sigmund, K. (2004). Evolutionary dynamics of biological games. Science 303, 793-799.

Parker, G. A. (1990). Sperm competition games: raffles and roles. Proc. R. Soc. London B 242, 120-126.

SAMUELSON, L. AND ZHANG, J. (1992). Evolutionary stability in asymmetric games. J. Econom. Theory 57, $363-391$.

SCHREIBER, S. J. (2001). Urn models, replicator processes, and random genetic drift. SIAM J. Appl. Math. 61, 21482167.

Skorokhod, A. V. (1989). Asymptotic Methods in the Theory of Stochastic Differential Equations. American Mathematical Society, Providence, RI.

TAYlor, P. D. And Jonker, L. B. (1978). Evolutionarily stable strategies and game dynamics. Math. Biosci. 40, $145-156$.

Weibull, J. W. (1995). Evolutionary Game Theory. MIT Press, Cambridge, MA.

Whittaker, J. C. (1996). The allocation of resources in a multiple-trial war of attrition conflict. Adv. Appl. Prob. 28, 933-964.

Whittaker, J. C. And Cannings, C. (1994). A resource allocation problem. J. Theoret. Biol. 167, 397-405. 